\title{
T Wave Abnormality by ECG Finding
}

National Cancer Institute

\section{Source}

National Cancer Institute. I Wave Abnormality by ECG Finding. NCI Thesaurus. Code C71083.

An electrocardiographic finding of a T wave which appears peaked, inverted, flattened or biphasic. 